\section{CORRECTION}

View Article Online

View Journal I View Issue

\section{A) Check for updates}

Cite this: Food Funct., 2022, 13, 459

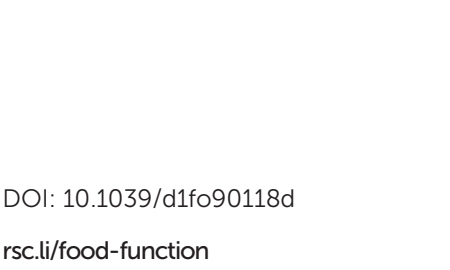

\title{
Correction: Enterobacter aerogenes ZDY01 inhibits choline-induced atherosclerosis through CDCA-FXR-FGF15 axis
}

\author{
Jinghui Tang, $\dagger^{\mathrm{a}, \mathrm{b}}$ Manman Qin, $\dagger^{\mathrm{a}, \mathrm{b}}$ Le Tang, ${ }^{\mathrm{a}, \mathrm{b}}$ Dan Shan, ${ }^{\mathrm{a}, \mathrm{b}}$ Cheng Zhang, ${ }^{\mathrm{c}}$ \\ Yifeng Zhang, ${ }^{a, b}$ Hua Wei, ${ }^{d}$ Liang Qiu* ${ }^{\star a, b}$ and Jun Yu*c \\ Correction for 'Enterobacter aerogenes ZDY01 inhibits choline-induced atherosclerosis through \\ CDCA-FXR-FGF15 axis' by Jinghui Tang et al., Food Funct., 2021, 12, 9932-9946, DOI: 10.1039/ \\ D1FO02021H.
}

The authors regret that there was an error with the author details in the original article. The first two authors should be marked as co-first authors.

The Royal Society of Chemistry apologises for these errors and any consequent inconvenience to authors and readers.

\footnotetext{
${ }^{a}$ Key Laboratory for Pharmacology and Translational Research of Traditional Chinese Medicine of Nanchang, Centre for Translational Medicine, Jiangxi University of Chinese Medicine, Nanchang 330006, China. E-mail: liangqiu@jxutcm.edu.cn; Fax: +86-791-87119895; Tel: +86-791-87119895

${ }^{b}$ Jiangxi Key Laboratory of Traditional Chinese Medicine for Prevention and Treatment of Vascular Remodelling Diseases, China

${ }^{c}$ Department of Physiology and Centre for Metabolic Disease Research, Lewis Katz School of Medicine, Temple University, Philadelphia, PA 19140, USA.

E-mail: jun.yu@temple.edu

${ }^{d}$ State Key Laboratory of Food Science and Technology, Nanchang University, Nanchang, Jiangxi, 330047, P. R. China

$\dagger$ These authors are co-first authors.
} 